\title{
Les étapes d'une dynamique de territorialisation : le pôle optique en Île-de-France
}

\section{The optics pole in the Ile-de-France Region : the stages of territorialization dynamics}

\author{
Elisabeth Decoster ${ }^{\mathrm{a} *}$, Andrée Matteaccioli $^{\mathrm{b} * *}$ et Muriel Tabariés ${ }^{\mathrm{c} * * *}$ \\ ${ }^{a}$ Ingénieur d'études CNRS, LTMU- UMR AUS, 4, rue Nobel, F - 77420 Champs-Sur-Marne \\ ${ }^{b}$ Maitre de conférences honoraire, Chercheur associé au MATISSE \\ Université Paris 1, 106-112, bd de l'Hôpital, F-75013 Paris \\ ${ }^{c}$ Ingénieur d'études Université, MATISSE, Université Paris 1 \\ 106-112, bd de l'Hôpital, F -75013 Paris
}

\section{Résumé}

Il s'agit d'expliquer la formation du pôle d'activités high tech dans le sud-ouest de la région parisienne, tout en reliant son fonctionnement à celui de l'ensemble de la région via les différents niveaux d'organisation du système régional d'innovation. Les concepts de «milieu innovateur» et de « cluster » seront utilisés pour décrire le fonctionnement de ce pôle, et plus particulièrement le pôle optique, dont les réseaux d'innovation se sont territorialisés récemment et dans lequel émerge un processus d'auto-identification.

* Auteur correspondant.

Adresse e-mail : elisabeth.decoster@univ-paris8.fr

** Adresse e-mail : andree.matteaccioli@wanadoo.fr

*** Adresse e-mail : tabaries@univ-paris1.fr 


\begin{abstract}
This paper aims to explain emergence of the high tech cluster in the south-west of Ile-de-France. Its functioning will be related to the rest of the Region through various organization levels of the regional innovation system. The concepts of «milieu innovateur» and «cluster » help to describe the functioning of the pole, and specially the « optics pole », of which innovation networks have been recently territorialized and in which a self-identification process is emerging.
\end{abstract}

Mots clés : Innovation, territoire, milieux innovateurs, «cluster », pôle optique, Île-de-France

Keywords: Innovation, territory, « milieux innovateurs », cluster, optics pole, île-de-France

Il s'agit de déterminer, dans une période où la compétitivité des territoires organisés est affirmée (Blanc, 2004), si des phénomènes d'ancrage territorial sont possibles dans une région métropolitaine comme l'Île-de-France. Si les logiques de localisation prévalaient à l'origine de la formation du complexe industriel de l'Île-de-France sud, il semble se dessiner aujourd'hui une logique de « milieu innovateur ». Cette hypothèse s' appuie sur les recherches et enquêtes ${ }^{1}$ menées régulièrement depuis une quinzaine d'années sur les trajectoires d'innovation des entreprises, les liens des entreprises innovantes avec leur milieu local et les centres de recherche, ainsi que le rôle des collectivités locales dans ce processus de territorialisation.

Elle sera plus particulièrement étayée par l'analyse du pôle optronique d'Île-de-France sud, connu maintenant sous le nom de «Vallée de l'optique », qui se présente comme un des nouveaux territoires de l'innovation en émergence dans une des zones les plus innovantes de la métropole parisienne.

De nombreux travaux émanant à la fois des économistes industriels, des géographes et des économistes territoriaux cherchent à expliquer le mode de fonctionnement et d'organisation de ces nouveaux pôles de l'innovation. Mais ils le font selon des grilles de lecture différentes. Pour les économistes industriels, l'entreprise est première et c'est au niveau de l'organisation industrielle qu'il convient de se placer pour comprendre la formation de ces territoires de l'innovation, car c'est la dynamique industrielle qui produit l'espace et lui confère ses caractéristiques. Pour les géographes, l'espace est premier et ce sont les grandes villes qui offrent une forme urbaine contextuelle favorable à l'innovation. Pour les économistes régionaux qu'il vaudrait mieux qualifier de « territoriaux », c'est la contextualité territoriale et le mode d'organisation du milieu local qui favorisent ou défavorisent les comportements innovateurs et le mettent en mesure de s'engager dans une dynamique créatrice.

\title{
1. L'approche industrielle : une approche évolutionniste de l'innovation, d'une conception a-spatiale à la prise en compte de l'espace et du territoire
}

\subsection{L'entreprise innovatrice structure l'espace}

Ce n'est que dans la première moitié du siècle dernier que les économistes se sont intéressés au problème de l'innovation. Jusque-là, ils la considéraient comme étant exogène au champ

\footnotetext{
${ }^{1}$ Voir en annexe la liste des enquêtes menées de 1985 à 2001.
} 
économique. J. Schumpeter fut le premier dans les années trente à la considérer comme partie intégrante de la fonction de l'entrepreneur en défendant la thèse que les grappes d'innovations entraînent des transformations de la structure industrielle. Contrairement aux classiques et aux néo-classiques qui appréhendent le temps comme celui du calendrier, Schumpeter raisonne dans un cadre temporel hétérogène. Les innovations portées par des entrepreneurs innovateurs sont à l'origine de la disparition d'entreprises obsolètes et de la création de nouvelles entreprises. Il considère ce phénomène de « destruction créatrice » comme étant au fondement même de la nature du capitalisme. En même temps, il intègre à son analyse la théorie des cycles longs de Kondratief pour expliquer que chaque grappe d'innovations qui se manifeste à travers une destruction créatrice donne lieu à un cycle de vie du produit, du procédé ou de l'organisation qui sur une période longue va évoluer vers la maturité et la sénescence.

Cette théorie évolutionniste du capitalisme qui met l'accent sur l'innovation ou plutôt sur les grappes d'innovations implique qu'au cours du déroulement du cycle, l'innovation se diffuse, mais ce n'est pas à proprement parler une diffusion spatiale : elle concerne seulement le marché au fur et à mesure que des entrepreneurs imitateurs y pénètrent, car elle est essentiellement liée à l'existence du profit. La diffusion dans l'espace national, voire international résulte de la décision des entrepreneurs de trouver de nouveaux espaces vierges pour que l'innovation puisse y produire des profits. L'innovation résulte dans cette approche uniquement de l'entrepreneur, qu'il s'agisse de l'entrepreneur individuel ou de la grande entreprise et sa diffusion dans l'espace ne se produira qu'aussi longtemps qu'il existera des innovateurs.

Selon cette approche, les nouveaux territoires de l'innovation en Île-de-France sud sont considérés comme de simples réceptacles d'entreprises innovatrices, notamment des équipementiers, qui trouvent des opportunités de profits sur le marché de l'instrumentation (pour le CEA, l'université scientifique d'Orsay, le CNRS, les grandes écoles, comme Polytechnique, Supélec, etc.). Les interrelations sont avant tout des relations de marché privilégiant la compétence plutôt que la proximité. L'accumulation de compétences attire des entreprises technologiquement avancées, grandes et petites, dans cette zone qui offre tous les facteurs de localisation avantageux pour les secteurs de haute technologie (armement, électronique, optique). Ainsi, selon cette approche, seules les entreprises innovatrices structurent l'espace, mais ce dernier n'offre que des facteurs favorables de localisation (bassin de main-d'œuvre qualifiée, accès aux centres de décision parisiens et à l'international, notamment en matière d'infrastructures, etc.).

Avec le concept de «pôle de développement» qu'il définit comme « une unité économique motrice ou un ensemble formé par de telles unités », Perroux (1964) va plus loin que Schumpeter : ce n'est plus simplement l'innovation qui déstructure-restructure son espace, mais c'est le processus de développement dans son intégralité qui est déstructeur-restructurateur et qui dure autant de temps que la firme motrice exerce une attraction sur son environnement. Ce processus est fondamentalement asymétrique, des processus cumulatifs de sous-développement à la périphérie se mettant en place parallèlement aux processus cumulatifs de développement dans le pôle. Sur la longue période, les phénomènes de diffusion l'emportent sur le déséquilibre de la polarisation, celle-ci exerçant des effets d'entraînement dans l'espace.

F. Perroux attire l'attention sur le fait que la phase de diffusion du développement ne peut être interprétée comme une phase d'égalisation étale dans l'espace (Perroux, 1964). 
Ce processus ne peut donc être assimilé à un processus d'uniformisation entre les deux espaces au cours de la phase de diffusion, mais il correspond à une double structuration, de nature différente, avec un décalage dans le temps.

\subsection{Une approche qui prend en compte l'espace et le territoire}

P. Aydalot ${ }^{2}$ va au-delà des thèses de la structuration de l'espace par l'entreprise motrice et de la division spatiale du travail ; il observe au milieu des années 70 un retournement des dynamismes spatiaux qui se manifeste d'un côté, par l'enlisement dans la crise des régions anciennement industrialisées et d'un autre côté, par l'émergence de nouvelles régions anciennement sous-développées, la plupart du temps vierges de toute industrialisation.

Il formule l'hypothèse que le changement technologique joue un rôle de premier plan dans ces processus de retournement, prolongeant la théorie du «cycle du produit » (Vernon, 1966, Markusen, 1985) selon laquelle un produit nouveau qui est conçu dans une région du monde, se diffuse dans des régions de plus en plus périphériques au fur et à mesure qu'il entre dans une phase de maturité. Ce processus de retournement est analysé comme une phase d'un cycle spatial, celle de l'émergence d'un nouveau paradigme technologique, dès que l'on prend en compte la longue période. À chaque période du cycle de l'innovation (émergence, maturité, rupture nouvelle) correspondrait un mode de structuration de l'espace (polarisation, diffusion, retournement, sans que ce dernier soit pour autant automatique pour tous les espaces).

La nouvelle polarisation spatiale liée à la phase de retournement technologique pose de nouvelles questions : «quels milieux, quels types de tissu industriel se révèlent les plus aptes à lancer de nouvelles activités, à internaliser les processus avancés, à diffuser en leur sein et au-dehors les innovations?» (Aydalot, 1985, p. 175). Avec la théorie du cycle spatial, une place éminente est accordée à l'espace territorial, puisque dans la phase de retournement, ce n'est plus l'entreprise mais le territoire qui innove. Le concept de «milieu innovateur » est proposé par P. Aydalot, qui distingue plusieurs logiques à la source de la création ou de la régénération des « milieux innovateurs » : la logique de la polarisation, la logique de l'attraction, la logique de la rupture-filiation, qui correspondent à des types de milieux différents. La première correspond plutôt à une forme métropolitaine ou technopolitaine où l'innovation procède de la connaissance, la seconde à une forme de type « sun-belt » ou région non industrielle et la troisième à une forme de type : ancienne région industrielle opérant une bifurcation à partir de ses anciens savoir-faire grâce à l'introduction de nouvelles technologies.

Cette approche territoriale de l'innovation va susciter chez les économistes industriels, mais aussi chez les géographes, des analyses fort contrastées. En économie industrielle, deux thèses s'affrontent, l'une selon laquelle l'organisation industrielle seule structure l'espace et l'autre pour laquelle l'environnement de l'entreprise doit être pris en considération en même temps que l'organisation de la dynamique industrielle.

\subsubsection{Une approche industrielle organisationnelle décontextualisée}

Les économistes industriels qui mettent en évidence l'importance de la constitution de réseaux d'innovation entre les grandes entreprises dans le contexte de la mondialisation,

2 Cf. l'ouvrage publié en 2004 sur l'œuvre de Philippe Aydalot aux éditions L'Harmattan par A. Matteaccioli. 
remettent en cause le concept même de milieu innovateur. Par exemple A. Rallet défend la thèse que «la territorialisation des processus d'innovation technologique » ne résulte pas « de la définition a priori du territoire comme agent actif de ces processus », mais plutôt de «la nécessité d'une relation de proximité entre les agents pour établir ces processus » (Rallet, 1993, p. 373). C'est l'organisation des firmes en réseaux qui favorise la proximité « organisationnelle» et non «spatiale» de ces agents. Les innovations sont fortement décontextualisées, surtout si elles sont directement fondées sur la science. Dans la même ligne, M-C. Belis-Bergouignan, G. Bordenave et Y. Lung se placent sur le terrain des dynamiques industrielles et soutiennent que «la complexité des interactions global-local se jouent dans l'espace de la firme » (1993, p. 529).

\subsubsection{Une approche industrielle organisationnelle qui tend à prendre en compte le contexte territorial}

Un autre courant d'économie industrielle introduit dans son analyse la notion de contexte et ne se place plus dans une problématique de localisation, mais de dynamique créatrice du territoire.

Cette idée était déjà présente dans les travaux de F. Perroux sur Schumpeter, quand il remettait en question la génération interne et autonome de l'innovation : «l'observateur des sociétés vivantes s'interrogera donc avec raison sur les circonstances qui président à la naissance de l'innovation. (Celle-ci) ne surgirait pas sans un milieu de civilisation, d'une part, sans un ensemble d'occasions, d'autre part, qui fournissent à l'innovateur ses instruments techniques et offrent à son regard le tableau des succès possibles. Comment ces candidats à l'innovation sont-ils préparés? comment le milieu leur accorde-t-il leurs chances?»(Perroux, 1965, 1993, p. 302).

La prise en compte de l'environnement de l'entreprise dans le cadre du processus d'innovation est plus particulièrement le fait des économistes industriels de «l'approche intégrée » qui, comme Gaffard (1986) mettent l'accent sur le processus d'innovation comme « création de technologie » et « comme processus de recherche et d'apprentissage aboutissant à l'apparition de qualifications entièrement nouvelles qui changent l'environnement lui-même» (Amendola, 1985). L'innovation majeure oblige les entreprises à reconsidérer leur rapport au territoire. La création de technologie suppose que l'environnement devienne une composante essentielle de l'innovation et que ses différentes ressources soient utilisées et combinées de manière à engendrer une nouvelle organisation productive localisée. Ces ressources deviennent alors «spécifiques ». L'entreprise contribue à créer son environnement en instaurant un réseau de relations partenariales avec de multiples acteurs locaux. Ainsi, « le système territorial entre dans le fait productif» (Gaffard, 1986).

La notion de « ressource spécifique » est également utilisée par l'École de la proximité. À partir de la notion de ressources et d'actifs génériques et spécifiques, ces auteurs (Colletis, Gilly, Pecqueur, Perrat, Zimmermann, 1997) posent «que la rencontre productive entre un espace et une firme peut revêtir trois configurations polaires : l'activation de ressources génériques, la spécification d'actifs et la construction territoriale». La première correspond à une «logique de localisation », la seconde à une «logique de territorialisation ou d'ancrage territorial » et la troisième enfin à une logique de «construction d'un territoire », qui «naît de la combinaison d'actifs spécifiques engendrant des ressources spécifiques (nouvelles) non exclusives ou non appropriables ». Le territoire apparaît dès 
lors pour eux « comme un «dispositif cognitif collectif», apte à réduire le risque de comportement opportuniste et à élargir le spectre des combinatoires productives potentielles ». Cette vision rejoint celle du GREMI ${ }^{3}$ et de J.-L. Gaffard, dans la mesure où l'apprentissage collectif est le pivot de la construction territoriale et de la dynamique techno-produtive : "En effet, le territoire est à la fois la résultante d'apprentissages passés et la condition des apprentissages futurs dans un processus dynamique inscrit dans le temps long » (ibidem, p. 128-130).

Le territoire apparait, non plus seulement comme un simple support de grandes entreprises innovatrices complexifiant leurs relations en multiples réseaux entre les « régionsmonde », mais comme un système local d'innovation au sein duquel les acteurs sont aptes à nouer des liens à la fois individuels et sociaux qui leur permettent de faire transiter des connaissances et des savoir-faire non codifiés qui ne sont pas transposables ailleurs, ce qui confère au territoire un avantage concurrentiel ; ce faisant, ces partenariats scientificoindustriels contribuent à générer un processus d'enrichissement des savoir-faire et des compétences, ce qui donne au territoire la possibilité d'avoir une capacité créatrice.

\section{L'approche urbaine : la métropole, une forme urbaine contextuelle favorable à l'innovation}

Pendant longtemps les études sur les relations entre la science, la technique et la ville furent rares. Ce n'est que tardivement que l'on trouve des études liant progrès technique et phénomène urbain et cherchant à cerner la composante urbaine de l'innovation (Pred, 1966, Schmookler, 1966). Hägerstrand (1967) insiste sur la notion de proximité et sur celle de contacts. Rogers et Shoemaker (1971) prennent en considération l'éducation et la mobilité sociale dans la diffusion de l'innovation.

Les analyses en termes de réseaux vont renouveler cette approche. Dans un monde de plus en plus globalisé, la métropole joue le rôle de centre, de nœud de réseaux, de pôle incubateur et attracteur. Les relations entre les métropoles, centres de commandement économique et financier à l'échelle de la planète, deviennent dominantes et éclipsent les relations traditionnelles (Sassen, 1991, 1996, Castells, 1996). Ces auteurs mettent en exergue le rôle des métropoles dans la connexion des réseaux de financement et des réseaux interfirmes. La métropolisation est alors appréhendée comme un processus de transformation.

Parmi ces transformations, il faut prendre en compte un accroissement accru des capacités cognitives de la métropole. Mais dans un monde où les connaissances deviennent rapidement obsolètes et où la concurrence est exacerbée sur les marchés, les entreprises ont un besoin permanent d'accéder aux informations les plus récentes aussi bien scientifiques, techniques, que sur l'évolution des marchés. L'accès direct à l'international est alors déterminant et renforce les nouveaux dynamismes innovateurs.

P. Veltz adopte largement cette grille de lecture. L'organisation métropolitaine joue un rôle central dans les différentes sortes et étapes de l'innovation actuellement. Le local est seulement "un élément ou plutôt un lieu de croisement de réseaux multi-échelles où se déploient de multiples logiques de proximité et de synergies, les unes territoriales, les

\footnotetext{
${ }^{3}$ Groupe de Recherche Européen sur les Milieux Innovateurs.
} 
autres non territoriales ». La métropole reste avant tout «le cadre fondamental (bien que non exclusif) des dynamiques actuelles de l'innovation »(Veltz, 1999, p. 606).

Cette approche par la métropole en tant que nœud de réseaux de métropoles rejoint la thèse des économistes industriels qui privilégient les collaborations scientifiques et techniques inter-firmes à l'échelle mondiale. Dans l'approche urbaine, la métropole constitue le contexte le mieux adapté pour innover dans un monde en changement. Par la multiplicité des possibilités d'échanges instantanés avec une multitude d'acteurs qui détiennent la connaissance, le pouvoir et la finance, non seulement à l'échelon national, mais international, la métropole constitue un contexte particulièrement favorable.

Malgré le handicap que constituent les dysfonctionnements liés à la fragmentation spatiale et sociale (Beckouche, Cohen, Damette et al., 1989, Aydalot, 1986a), la métropole apparaît cependant bien comme la forme majeure favorisant tous les types d'innovation (scientifique et technique, culturelle, financière), comme «l'espace assurantiel» par excellence, qui permet à la fois aux entreprises et aux salariés de supporter les à-coups conjoncturels (Veltz, 1993).

Les études urbaines ont fait apparaître d'abord, la métropole urbaine dans son ensemble, comme un système favorable à l'innovation, puis ont permis de mettre en évidence la complexité des espaces métropolitains. Les études, menées dans une problématique de milieu innovateur, ont fait apparaître qu'au sein de la métropole on pouvait identifier différents niveaux d'organisation en matière d'innovation.

De la même façon qu'on observe des différenciations socio-spatiales au sein de la métropole, on a pu observer au sein de la métropole parisienne une différenciation des dynamiques d'innovation selon les acteurs et les espaces concernés. Trois niveaux d'organisation ou « milieux innovateurs » ont été distingués, qui vont du plus global au plus local, formant ensemble le système régional d'innovation (Decoster, Matteaccioli, Peyrache, Tabariés, 1993) :

- le «milieu métropolitain », dont le mode dominant d'innovation est « la veille et la stratégie technologique » et identifiable à travers les relations d'acteurs à logique extra-territoriale, fortement polarisés dans l'« hyper-centre » parisien ;

- les «micro-milieux » s'organisant en technopôles, liant entreprises de haute technologie et centres de recherche, orientés vers la «création technologique »;

- et le «milieu industriel diffus » ayant peu de cohésion interne et une faible polarisation spatiale, et où dominent les logiques «d'exploitation » ou de « développement technologique ».

Alors que le milieu métropolitain est très lié au national dans un pays centralisé comme la France, l'échelle de la régulation des activités scientifiques et technologiques étant la nation, les micro-milieux et le milieu diffus sont à l'échelle régionale-locale ; c'est le niveau des interactions complexes (liées à l'informel, au tacite), l'échelle de la formation, du recrutement, de l'interdisciplinarité. Les micro-milieux sont le support de la technopole en ce sens qu'ils fonctionnent à la fois à un niveau local/régional et à un niveau métropolitain. L'articulation de ces différents niveaux est loin d'être réalisée, seuls les réseaux de création technologique animés par les centres de recherche publics semblant capables de relier niveau métropolitain et niveau du tissu diffus, via le niveau technopolitain : en effet, l'acteur de recherche public est à la fois présent dans les réseaux de veille, de création et de développement technologique. 
L'analyse s'est concentrée sur ces nouveaux territoires innovants de l'Île-de-France sud, afin d'identifier, à côté d'une logique de localisation métropolitaine, une logique de territorialisation entrant pour une part croissante dans l'explication du dynamisme et de la capacité créatrice de ces territoires et plus particulièrement du pôle optique. Une meilleure compréhension des processus en œuvre conduit donc à se tourner vers l'approche territoriale.

\section{L’approche territoriale : la dynamique créatrice des territoires}

Au milieu des années 80, P. Aydalot marquait les limites d'une pensée, quasiment partagée par tous les économistes de l'époque, selon laquelle l'innovation était supposée venir exclusivement de l'extérieur et être diffusée par les grandes entreprises.

«On peut avoir de l'innovation une vision plus territorialisée ; l'innovation est alors la création d'un milieu pour répondre à un défi ou à un besoin local par l'utilisation de l'expérience locale : elle est le fruit de l'inventivité du milieu et répond aux besoins du développement local, moyen que se donne une société pour progresser... (Aydalot, 1986b, p. 57). L'attention se focalise moins sur les conditions de diffusion du progrès technique que sur ses conditions territoriales d'émergence : «La créativité est toujours enracinée dans l'expérience et la tradition. Les connaissances accumulées constituent toujours la base du progrès. La créativité exige la rencontre, le face à face inorganisé, une certaine spontanéité que les grandes entreprises où toute chose est programmée, ne peuvent fournir; elles constituent le contraire d'une structure innovatrice. Ici encore, la petite échelle, les milieux de petite dimension, l'absence de zonage, de spécialisation des lieux et des hommes sont nécessaires » (Aydalot, 1985, p. 148). "L’hypothèse a donc été faite du rôle déterminant joué par les milieux locaux comme incubateurs de l'innovation, comme prisme à travers lequel passeront les incitations à l'innovation et qui donnent sur le terrain son visage à celle-ci ; l'entreprise n'est pas un agent innovateur isolé ; elle est partie du milieu qui la fait agir. Le passé des territoires, leur organisation, leurs comportements collectifs, le consensus qui les structure sont des composantes majeures de l'innovation... ce ne sont pas les entreprises mais les milieux locaux qui innovent » (Aydalot, 1986c, p. 11).

L'émergence de nouveaux territoires de l'innovation en Île-de-France sud au sein desquels l'innovation provient souvent de la science relève d'une logique de la polarisation technopolitaine. Mais cette polarisation peut procéder elle-même de deux types de logiques:

- une logique classique de localisation qui caractérise encore l'ensemble de la zone high-tech de l'Île-de-France sud, malgré l'apparition récente de phénomènes d'endogénéisation dans son tissu industriel ;

- ou une logique de création territorialisée qui émerge dans le pôle optronique, en raison de l'apparition en son sein de trois propriétés nouvelles qui interagissent entre elles : une logique organisationnelle de mise en réseaux, une logique cognitive d'apprentissage, une logique territoriale. Ce sont là les caractères génériques des milieux innovateurs, tels qu'ils ont été mis en évidence par le GREMI (Quévit, Van Doren, 1997). En définitive, le milieu innovateur est «un ensemble territorialisé dans lequel des interactions entre agents économiques se développent par l'apprentissage qu'ils font de transactions multilatérales génératrices d'externalités spécifiques à l'innovation et par la convergence des apprentissages vers des formes de plus en plus performantes de gestion en commun des ressources » (Maillat, Quévit, Senn, 1993, p. 6). 


\section{Les dynamiques de territorialisation en Île-de-France sud}

Des indices du passage d'une logique de localisation à une logique de territorialisation des dynamiques d'innovation dans un territoire métropolitain innovant, l'Île-de-France sud, sont repérables à travers l'observation des réseaux de coopération entre science et industrie qui s'y développent sur le long terme, des relations inter-entreprises et des phénomènes d'essaimage. Du fait du contexte métropolitain, très prégnant en Île-de-France, et du rôle de l'État dans cette région et plus particulièrement dans le complexe militaro-industriel de l'Île-de-France sud, on peut d'ores et déjà postuler qu'il s'agira d'un type de milieu innovateur assez particulier, dont la relation au territoire sera plus lâche et le branchement sur les réseaux internationaux plus important.

\subsection{D’une zone vouée à la recherche à une zone technopolitaine}

À l'origine, l'île-de-France sud apparaît comme une zone vouée à la recherche et à l'enseignement supérieur, installés sur ou autour du plateau de Saclay par la volonté étatique à partir des années 50, puis comme une zone de desserrement des entreprises dans les décennies suivantes. Celles-ci ont été principalement attirées

- par la réalisation de zones industrielles modernes proches des infrastructures routières et aéroportuaires et au cœur des villes nouvelles d'Évry et de Saint-Quentin-en-Yvelines,

- par la proximité de lieux résidentiels valorisés,

- par la présence de centres de recherche, à la fois clients et fournisseurs de connaissances scientifiques.

On assiste aussi à la lente construction de l'état d'esprit technopolitain, qui commence avec les acteurs de la recherche (AES ${ }^{4}$, Île-de-Science), pour se poursuivre avec les collectivités locales (conseil général de l'Essonne) et enfin les grandes entreprises (en particulier celles impliquées dans le pôle optique).

\subsubsection{Constitution d'un potentiel scientifique très important autour du plateau de Saclay}

Le rôle de l'État et des groupes a été structurant dans l'émergence de ce complexe scientifique et industriel. La recherche publique et privée a joué véritablement un rôle d'attracteur.

Cette concentration scientifique unique en France regroupe aujourd'hui la plupart des domaines scientifiques, mais quelques-uns sont particulièrement importants, par la qualité des équipes de recherche et par les potentialités de développement industriel qu'ils recèlent : l'informatique, les mathématiques, l'optique, l'instrumentation physique et les lasers, la physique nucléaire, les biotechnologies, les nouveaux matériaux et l'agroalimentaire.

\subsubsection{D’une logique de desserrement depuis Paris}

et la proche banlieue à un développement endogène du tissu économique

Si le développement est en partie celui d'une périphérie métropolitaine, obéissant à une logique de desserrement et à une fonctionnalisation de l'espace métropolitain, il correspond par ailleurs aussi à la formation d'un complexe industriel, et à un processus de polarisation autour de la haute technologie. On a pu observer dans cette zone en quelques années un foisonnement de PME tertiaires et industrielles, du fait des réorganisations inter-

\footnotetext{
${ }^{4}$ Voir en annexe la liste des sigles utilisés.
} 
nes des grands groupes (externalisation, filialisation et recentrage sur le métier) et de la mise en place de nouvelles conditions techno-productives qui ont favorisé la création de PME, innovant sur des créneaux pointus et se développant selon une stratégie de grappe.

Plusieurs vagues de création d'entreprises ont alimenté la dynamique de développement local. Une première vague dans les décennies 1950-60, en grande partie dans la mouvance du Centre d'Études Nucléaires, a été celle des entreprises sous-traitantes créées par des catégories ouvrières (ouvriers qualifiés et contremaîtres).

Une deuxième vague de «PMIsation » s'est réalisée au cours des décennies 70 et 80 par attraction et essaimage local, principalement dans les activités électroniques, informatiques et d'instrumentation. Un nouveau type d'entreprises a émergé : entreprises dont le projet industriel est innovant et technologique, entreprises engagées dans des relations de sous-traitance plus complexes, qui se transforment en co-traitance et qui exigent des chefs d'entreprise ayant un savoir-faire technique important. Ces nouvelles relations inter-entreprises ont accru considérablement l'utilité d'une proximité géographique et ont donc constitué un facteur de concentration des entreprises dans la zone (Decoster, Tabariés, 1992).

La polarisation spatiale qui s'est réalisée le long de l'axe sud correspond en définitive à un tissu productif diversifié et inter-relié, composé de segments de groupes industriels dans les activités de conception, de petites entreprises industrielles ou de services introduisant ou adaptant de nouvelles technologies, et de centres de recherche publics.

Les dynamiques d'innovation des entreprises motrices ont entraîné le développement de certains types de firmes : activités de biens d'équipement et intermédiaires très spécialisés, en particulier dans l'instrumentation (scientifique, militaire, médicale...), de petites firmes de haute technologie très pointue, de sous-traitants de haute technicité. La sous-traitance en cascade, particulièrement développée dans les secteurs de l'électronique et de l'aéronautique, mais qui est aussi une pratique courante des PMI high tech qui externalisent tout ce qui n'est pas leur savoir propre, a favorisé le développement d'un tel tissu industriel. Elle a constitué aussi un facteur de diffusion intersectorielle du dynamisme dans la mesure où ce processus intègre des petites entreprises de secteur traditionnel, en particulier de la mécanique.

On retrouve dans ce tissu les spécialisations sectorielles impulsées par les grands programmes technologiques nationaux qui ont modelé les «pôles de compétitivité » français (Aglietta, Boyer, 1983), et plus particulièrement l'Ile-de-France qui a bénéficié à plein de ce « colbertisme high tech » (Cohen, 2002). Son système d'innovation est basé sur quelques secteurs productifs, comme les télécoms, l'énergie, l'électronique, l'aéronautique et le spatial, et apparaît finalement assez cloisonné, vertical et peu diffusant (Serfati, 1994), ce qui a constitué un handicap conséquent pour la diffusion intersectorielle.

\subsection{Les évolutions récentes autour du plateau de Saclay: montée en puissance des collaborations recherche-industrie}

Jusqu'à la fin des années 90, les stratégies technopolitaines de mise en réseau sont surtout le fait des acteurs publics (État, laboratoires, collectivités locales), puis, avec le changement d'attitude des acteurs privés, en particulier des grands groupes présents dans la zone, s'opère une sorte de révolution qui voit ces entreprises s'investir dans la territorialisation des compétences ${ }^{5}$.

5 Cf. enquêtes 1985, 1987, 1990, 2000 et 2001. 


\subsubsection{Une valorisation des compétences des centres de recherche en plein essor}

Les grands programmes technologiques nationaux ont orienté et marqué non seulement la structure de l'appareil productif, du potentiel scientifique avec ses grands organismes sectoriels de recherche finalisée dans le nucléaire (le CEA), les télécommunications (le CNET), le spatial (le CNES), mais aussi la nature et les formes des relations entre recherche et industrie.

En Île-de-France sud, deux grands types de logique techno-économique ont été à l'origine des réseaux de collaboration et de structuration des milieux, la première autour du Centre d'Études Nucléaires de Saclay, correspondant à une dynamique de valorisation de la recherche du type «externalisation et transfert des résultats », et l'autre plus complexe à partir des centres de recherche et d'enseignement supérieur correspondant à une dynamique de « valorisation des compétences ».

A. Des réseaux de relations essentiellement animés par les grandes entreprises et le Centre d'Études Nucléaires dans les années 80

Au cours de cette période, le cloisonnement des équipes de recherche universitaires est encore important; leur contribution à l'innovation industrielle reste faible, car elle demeure cantonnée à des réseaux de veille technologique et scientifique avec les centres de recherche des grandes entreprises et ne représente qu'un apport ponctuel de compétences isolé de toute préoccupation industrielle. Une communauté sociotechnique et des réseaux technico-économiques se sont intensément développés à partir des grands programmes technologiques. Ils ont concerné de façon privilégiée les laboratoires de recherche et les directions de R\&D des grandes firmes qui appartiennent aux secteurs industriels bénéficiaires de ces programmes, et qui ont plutôt développé des stratégies de « cueillette».

Les réseaux autour du CEN sont issus d'une part de la politique générale du CEA dont l'objectif est d'externaliser certaines de ses activités, et de s'appuyer sur la sous-traitance pour se recentrer sur ses domaines de compétences, et d'autre part, ils ont résulté d'une politique de transfert de technologie et de la valorisation de la recherche nucléaire dans des applications civiles. Cette dernière politique a visé à encourager l'essaimage et à développer des relations partenariales avec l'industrie.

Le développement important dans les années 80 du second type de relations a fait du CEN un des principaux acteurs du milieu. L'activité de transfert technologique a mobilisé, dans le cadre de «cellules de valorisation », de nombreux chercheurs et techniciens et a concerné, bien au-delà du nucléaire, tant des technologies de pointe (microélectronique, robotique, matériaux...) que des domaines avancés de secteurs plus traditionnels (mécanique, génie chimique...). Offre d'informations, conseil technologique, formation, aide à la création d'entreprises ont été au cœur de partenariats et de réseaux contractuels bilatéraux entre industrie locale et CEN, qui a joué un rôle leader.

Les effets attendus sur le milieu de ce type de politique de valorisation sont d'en accrô̂tre la capacité d'innovation, de qualifier les ressources humaines locales et de diversifier le système productif en lui ouvrant l'accès à de nouveaux marchés. Mais outre qu'elle présuppose une bonne qualité et une diversité du tissu industriel environnant lui permettant de saisir les opportunités de développement offertes, elle est une stratégie d'offre à des entreprises qui ne sont pas insérées dans son réseau «naturel ». 
Or, que ces partenariats concernent le transfert technologique pur (exploitation sur de nouveaux marchés d'innovations et de produits mis au point par le CEN) ou le développement technologique (développement d'applications non nucléaires à partir des résultats du CEN et avec son aide technique), on constate d'une part que les entreprises partenaires ont plutôt été celles qui étaient déjà impliquées dans des relations de sous ou de co-traitance avec le CEN, car celles-ci ont permis de constituer un capital relationnel, élément particulièrement important dans le secteur nucléaire peu ouvert sur l'extérieur.

D'autre part étant donné la faiblesse de l'essaimage et le contenu technologique très avancé des transferts potentiels, ces réseaux du CEN ont été largement extra-locaux. Cette politique de gestion d'un stock d'innovations potentielles à la recherche de marchés et d'industriels, qui « propose des réponses et recherche ensuite les questions », est tout à fait représentative des politiques technologiques qui ont été menées au niveau local au cours de cette période en France. Elle correspond à une vision du développement technologique sur le modèle du marché, de la combinaison de ressources, dont il faut aménager au mieux l'offre sur un marché qui se révèle défaillant. Dans une phase ultérieure et sur un mode très différent de cette stratégie d'exploitation et de court terme, des établissements académiques ont tenté de déployer de nouvelles stratégies de création de PME de haute technologie, stratégies de long terme et de création, mettant l'accent sur une valorisation dynamique basée sur la création permanente de nouvelles compétences.

B. La montée des coopérations entre recherche et industrie dans les années 80-90:

le rôle des laboratoires académiques

On a pu observer en quelques années un développement très important des collaborations industrielles des laboratoires publics avec des PMI et l'émergence d'un « ancrage local » de ces partenariats. Ce sont les équipes universitaires qui ont été le plus touchées par ce phénomène (liens procurés par la formation, et nécessité de ressources financières supplémentaires). Parallèlement on a observé une implication de plus en plus forte des industriels dans les formations universitaires (IUT, DEA, création de filières de formation spécifiques, de chaire professorale, de centre d'enseignement ${ }^{6}$ etc.). Des activités d'expertise et de services dirigées vers les entreprises locales se sont développées, en particulier à l'université d'Orsay, mais le cas de figure dominant cependant reste celui de l'intégration des chercheurs aux faisceaux d'experts constitués par les grandes entreprises pour leur veille technologique.

De nouvelles formes de relations entre recherche et industrie se sont développées, grâce aux évolutions culturelles initiées par les politiques de la recherche du début des années 80 : échanges de personnel, financements de doctorants, échanges scientifiques informels, partenariats au sein de groupements ${ }^{7}$, coopérations avec les entreprises essaimées, montages de structures associatives de recherche, de concertation, de transfert (Decoster, Tabariés, 1992). On a constaté également une forte augmentation des budgets de contrats industriels dans la plupart des laboratoires. Plusieurs types de réseaux d'innovation ont été mis en évidence par nos enquêtes, se différenciant selon l'intégration de l'équipe de recherche

\footnotetext{
${ }^{6}$ C.E.E.T.A.M., Centre d'Études et d'Enseignement des Technologies Appliquées aux Micro et nano-structures avec trois entreprises locales (IBM, HP et Thomson).

${ }^{7}$ Au début des années 90, de nombreux Groupements De Recherche du CNRS avec des sociétés privées impliquent des équipes de recherche publique de la zone d'étude : 16, dont 3 en optique.
} 
dans le processus d'innovation, les méthodes de travail, de concertation et le niveau de « recomposition » opéré (Decoster, 1991) :

- la collaboration bilatérale classique coupée de toute préoccupation industrielle (veille ou apport extérieur de compétence),

- le «pseudo-partenariat » ou « quasi-partenariat» de recherche technologique de base (veille, apport extérieur et groupements scientifiques du CNRS) où l'intégration est plus importante entre les partenaires,

- le partenariat technologique de base où l'intégration est plus poussée encore avec une combinaison des compétences (exemple du «partenariat bilatéral organique », quand l'entreprise est issue de la recherche, des micro-réseaux locaux d'innovation, des réseaux européens où les équipes de recherche d'Orsay sont pleinement impliquées et qui promeuvent une nouvelle organisation en réseaux de la recherche, dans une logique plus « horizontale » (Callon, Laredo, 1990)...).

L'enquête a confirmé l'importance des réseaux informels et le rôle de la formation comme source de ces liens informels qui sont à la base de la très grande majorité des coopérations entre l'industrie et la recherche. On a assisté par ailleurs au début des années 90 à la mise en place de dispositifs incitatifs au niveau local, avec la politique du conseil général de l'Essonne, très novatrice en matière de soutien à la recherche. En particulier le soutien à la création d'instituts (de biotechnologie des plantes, des matériaux), dans la mesure où ils ont des liens avec le tissu industriel local, vise à repositionner la recherche, dans une démarche de pôles et de transfert.

Cette constatation du rapprochement entre recherche et industrie est confortée par la dernière enquête menée (Cohen, Decoster, Tabariés, 2001) auprès d'entreprises (grandes entreprises innovant dans le cadre d'un projet européen BRITE et PMI innovant en dehors de ce cadre) de la zone technopolitaine. Celle-ci a évidemment confirmé que les facteurs de localisation premiers étaient le marché du travail très qualifié, l'accès à Paris, le bon réseau de transport (dont l'aéroport), mais elle a fait apparaître un facteur second, la proximité des collaborateurs et des universités. Pour toutes les firmes, les contacts avec les établissements d'enseignement supérieur et de recherche sont importants, même si les réseaux d'affaires (clients, fournisseurs, concurrents, services aux entreprises) sont encore plus importants. Enfin, en ce qui concerne la localisation des partenaires, les concurrents locaux proches sont plus nombreux que les clients et les fournisseurs, ce qui donne corps à l'hypothèse de l'émulation par la concurrence (Porter, 1998, 2000). Il faut signaler que du fait de la dimension européenne intrinsèque des réseaux BRITE, l'importance des liens locaux dans le milieu est probablement sous-estimée par ce type d'étude.

En définitive, la valorisation des compétences en Île-de-France sud recouvre deux modèles de dynamique technologique, très différents dans leur façon de s'appuyer sur le couplage formation - recherche fondamentale - recherche technologique, l'un que l'on peut qualifier de stratégie d'excellence, l'autre de stratégie de continuité technologique, essayant de combiner excellence et diffusion technologique.

a) La «valorisation d'excellence » des compétences correspond à une politique technologique et industrielle très ciblée, qui est le fait des équipes de recherche fondamentale, tant à l'université d'Orsay qu'à Polytechnique. Elle vise à renforcer et à ouvrir les réseaux d'innovation aux entreprises de haut niveau technologique. Les modalités 
en sont la création de sociétés de services pour des transferts de très haut niveau technologique, la formation d'ingénieurs par la recherche, les partenariats, les structures coopératives de recherche technologique de base, l'incubation de nouvelles entreprises. De telles actions et projets relèvent d'une conception anglo-saxonne de la science, qui est source et moteur du développement économique, et d'une stratégie de valorisation dynamique, par la formation et la création de compétences, afin de stimuler et de renouveler le tissu industriel. La priorité n'est pas la diffusion du dynamisme technologique à l'ensemble du tissu productif, local ou national, mais la réalisation des seules avancées technologiques. «Ouvrir la recherche à l'industrie et ouvrir l'industrie à l'excellence », une telle formule illustre bien cette stratégie de dynamisation de l'industrie, par l'excellence et l'élitisme.

On peut se poser la question de savoir si elle peut contribuer à réduire significativement le handicap typique de l'industrie française, à savoir la faible intégration de la recherche à la stratégie des firmes ? Ou ne fait-elle que reproduire et même amplifier la coupure entre deux catégories de firmes, celles aptes à la recherche, qui resteront très minoritaires, et celles qui y sont totalement étrangères? Si le second terme de l'alternative est le plus probable, alors ce type de politique ne fait que déplacer une frontière mais ne contribue pas à l'estomper, en ciblant seulement certaines catégories de firmes, en accroissant l'élite des entreprises innovatrices et la qualité de leurs réseaux.

b) À l'opposé de cette stratégie d'excellence, une stratégie de «continuité technologique » se développe dans des écoles d'ingénieurs plus professionnelles (ENSIA, Sup'Optique), et elle constitue un moyen de résoudre l'apparente contradiction entre une politique d'excellence de la recherche et des politiques locales de développement. Elle permet de créer des liens entre des partenaires non technopolitains et des partenaires «technopolitains» (PME de haute technologie et centres de recherche-formation), et peut ainsi faire bénéficier l'ensemble du tissu économique des externalités de la technopole. La continuité entre recherche fondamentale et applications industrielles caractérise les relations de ces écoles avec le monde économique. Cette dynamique de création-diffusion technologique et de développement local en émergence articule plusieurs éléments :

- diffusion technologique vers les industries traditionnelles, qui n'est pas un simple transfert puisqu'elle nécessite une recherche technologique de haut niveau menée en partenariat,

- rôle d'interface des PMI de haute technologie dans ce passage des innovations depuis les industries de pointe vers les industries traditionnelles et vers les PMI,

- continuum technologique au sein, ou auprès, de la grande école d'ingénieurs qui va de la recherche fondamentale au transfert de technologie,

- ouverture des réseaux d'innovation et de transfert initiés dans le milieu local sur la région, la France et l'Europe,

- soutien de pouvoirs publics locaux.

Cette simple énumération suffit pour comprendre que le développement d'une telle stratégie est nécessairement resté limité ; d'une part, fondamentalement elle implique de « révolutionner» le système technologique et la culture technique, de repenser l'ensemble de la chaîne de diffusion scientifique et technique, ce à quoi le milieu francilien est loin d'être propice, et d'autre part les initiatives les plus récentes concernant l'optique tendent 
« spontanément » à tirer la recherche vers la stratégie d'excellence plus que vers celle de la diffusion.

\subsubsection{Le processus de constitution du milieu de l'optique : émergence d'une logique de création territorialisée}

Au début des années 90, plusieurs « micro-milieux » sont ou déjà constitués ou en émergence en Île-de-France sud, parmi lesquels le pôle optique. Ils se caractérisent par la présence et l'importance des acteurs de recherche, des activités de conception des grandes entreprises et des PMI de haute technologie, dans des secteurs comme l'instrumentation (scientifique, militaire, médicale...), les télécoms, l'énergie, l'électronique, l'aéronautique et le spatial. Ces « sous-systèmes » sont relativement homogènes en ce sens qu'ils regroupent des PMI quelque peu semblables par leur profil (utilisations très pointues et très spécifiques des technologies), leur trajectoire technologique, la nature de leurs marchés (protégés ou captifs, souvent militaires); ils ont de ce fait une logique productive et entrepreneuriale très particulière, fondée sur la très haute qualité pour des petites séries ou du sur-mesure, ce qui les rend moins aptes à explorer de nouveaux champs d'activité, mais plus aptes à collaborer. L'ensemble a tendance à vivre en circuit fermé, et à s'engager dans des partenariats avec la performance technologique comme finalité commune.

Une dynamique endogène de micro-milieux de la haute technologie a eu tendance à se développer, confortant l'homogénéité d'un système d'innovation intégré aux "pôles de compétitivité » franciliens et au complexe militaro-industriel. Proximité organisationnelle et proximité spatiale se sont parfois confondues, comme pour le micro-milieu de l'instrumentation et de la «photonique» (mélange d'électronique, d'informatique, d'optique), que nous appellerons par commodité «pôle optique », constitué sur la base d'une importante et ancienne concentration spatiale d'organismes de formation et de recherche spécialisés, d'entreprises d'instrumentation et d'optique ainsi que d'entreprises utilisant l'optoélectronique, et ayant toutes un très haut niveau technologique.

Ce pôle optique est particulièrement significatif du processus qui s'engage et génère une véritable dynamique créatrice du territoire. On peut distinguer deux périodes : une première période d'émergence de 1980 à 1990, une seconde période de renforcement du micro-milieu de 1990 à nos jours, à travers des restructurations.

\section{A. Le rôle déterminant des PME de haute technologie}

et du partenaire universitaire dans les années 1980 à 1990

Un rôle déterminant a été joué par l'Institut d'Optique Théorique et Appliquée (IOTA), du fait de sa bonne intégration dans la communauté socio-professionnelle liée à sa stratégie de « continuité technologique », et par des PME en phase, qui ont joué un rôle moteur dans la transversalisation des technologies et permis la diffusion de l'innovation. Ces acteurs clés ont été les « passeurs » au sein du milieu.

En effet, au-delà des caractéristiques communes que nous venons de voir, le pôle optique a bénéficié dès les années 1980 d'un certain nombre d'opportunités. Ainsi, l'absence d'un centre technique professionnel pour cette industrie a conduit les PME de l'optique à se tourner, via le syndicat patronal de l'optique (GIFO), vers l'Institut d'Optique Théorique et Appliquée, qui a rempli la fonction d'un centre technique professionnel (résolution de problèmes d'atelier, de normes, de procédés, de contrôle de qualité). Cette collaboration 
entre le GIFO et l'IOTA, ainsi que l'embauche locale d'ingénieurs optroniciens formés à Sup'Optique, l'école d'ingénieurs de l'Institut d'Optique, ont permis au IOTA de jouer un rôle efficace dans le développement des réseaux de l'optique.

Outre les nombreuses collaborations de recherche entre équipes ${ }^{8}$ de recherche fondamentale spécialisées en optique et en physique, électronique et informatique, différents types de réseaux d'innovation ont contribué à structurer le pôle optique (réseaux de recherche coopérative, réseaux avec firme leader, réseaux plus collectifs, réseaux européens...) (Decoster, 1991).

Des initiatives locales ont tenté de conforter cette structuration. Depuis 1989, des concertations, à l'initiative de Thomson-CSF, entre PME, systémiers, recherche et administration se sont donné pour but l'identification des verrous technologiques de l'industrie optique et leur dépassement. Cette concertation a débouché en 1990 sur des solutions collectives dans différents domaines et sur des projets de recherche associatifs réunissant des partenaires pour la plupart localisés dans la technopole. Elle a également donné lieu à la mise en place à l'université d'Orsay d'une nouvelle formation en optoélectronique privilégiant l'approche système et à la création d'un laboratoire industriel à l'Institut d'optique d'Orsay. Deux acteurs sont à l'époque les plus dynamiques en ce qui concerne la structuration du milieu : l'IOTA et le groupe Thomson-CSF, qui s'est investi dans un processus d'ouverture sur son milieu et de coopération tous azimuts, appuyés par le groupement professionnel GIFO, qui a imprimé à ces initiatives un caractère collectif et associatif.

Ce « micro-milieu » est aussi caractérisé par ses PME de haute technologie, en particulier les PME d'instrumentation dont le grand savoir-faire technologique leur a permis d'être à la jonction de la recherche fondamentale et de l'industrie et de jouer un rôle très important dans la diffusion des technologies innovantes vers de nouveaux secteurs. L'ensemble de ces acteurs formaient au début des années 90 un milieu relativement communiquant, propice à la coopération, à l'intégration industrie-recherche. Le marché local du travail, les trajectoires de formation et les trajectoires professionnelles des individus ont joué un rôle essentiel dans la constitution d'un capital relationnel et d'une communauté, où les synergies ont été favorisées par la proximité spatiale.

Cependant ce type de «micro-milieu » reste très dépendant des décisions des grandes firmes leader et des décisions budgétaires de l'État en matière de recherche et de défense nationale, ce qui l'a fragilisé au cours de la décennie 90. Les éléments de stabilisation sont venus plutôt de la masse critique de compétences, de savoir-faire créés localement, et aussi des apprentissages, des pratiques de coopération développés dans les réseaux, et qui sont spécifiques au milieu local.

B. Maintien et renforcement du milieu :

les restructurations internes au cours des années 1990-2000

Alors que la structuration du système local d'innovation de l'optique devenait un enjeu majeur dans un contexte mondial de très forte croissance des marchés des technologies optiques jusqu'en 2001, la transformation des structures productives locales était profonde : tissu ancien plongeant dans la crise avec le basculement dans une situation où le marché civil est devenu prédominant et régénération du milieu s'opérant sur de nouvel-

\footnotetext{
${ }^{8}$ IOTA, Polytechnique, Paris XI, ENSTA, CNES, CNET, ENS, CEN de Saclay.
} 
les bases, avec des entreprises de très haut niveau technologique et aptes à se positionner sur des marchés mondialisés très concurrentiels (Cohen, Decoster, Tabariés, 2002).

Le déclin en tant que milieu du tissu industriel ancien s'est opéré dans la mesure où les relations de proximité des entreprises «traditionnelles » qui étaient la « règle » avec leurs partenaires (clients, sous-traitants, fournisseurs) se sont affaiblies. La proximité est devenue de moins en moins importante pour les relations industrielles classiques, avec l'adaptation à de nouveaux marchés et le contexte concurrentiel accru. Une nouvelle norme s'est imposée, celle de la concurrence ${ }^{9}$.

Par ailleurs le renouvellement du tissu des PME s'est réalisé par la création de nombreuses start up, fortement liées au milieu de la recherche et des grandes entreprises. Le milieu essonnien a participé pleinement de cette dynamique qui a concerné l'ensemble de la France. Ainsi, au niveau de la technopole ${ }^{10}$, on observe une augmentation assez forte des effectifs $(+22 \%)$ et des établissements $(+9,8 \%)$ entre 92 et 98 dans le secteur de l'instrumentation-optique (NAF 33) tandis qu'elle décroissait en France et en Île-de-France ${ }^{11}$.

Dernier élément de transformation du milieu : le repositionnement des établissements des grands groupes, tel Alcatel et Thomson-CSF (devenu Thales), SFIM, CILAS, et d'importantes PME high tech telles que Micro-Contrôle, Jobin-Yvon et Quantel. Ces divisions optiques de grandes entreprises ont connu des restructurations très importantes au cours des dernières années, restructurations qui ont entraîné des modifications des stratégies d'innovation de ces groupes, et, nous en faisons l'hypothèse, de leur inscription territoriale en Île-de-France sud.

Ainsi, à l'égard des petites entreprises, les grands groupes ont abandonné leur stratégie de liquidation-absorption. Les nouvelles politiques d'alliance, de capital-risque, correspondent à une reconnaissance des avantages spécifiques de la PME en matière d'innovation, de la fonction d'interfaçage qui peut être la sienne. Les technologies optiques sont typiquement des technologies de rupture qui nécessitent une grande réactivité et une légèreté des structures, que seules les start up et les PME possèdent. Elles sont encore mal maîtrisées, relativement récentes et ont donc un potentiel important d'idées nouvelles fondamentales. Du fait qu'elle est transverse et diffusante, l'optique a une forte capacité à coller aux marchés émergents et en forte croissance. Ce type d'innovations permet une redistribution du jeu en annulant les rentes de situation, et le raccourcissement des cycles, tant des technologies que des produits, qui se comptent en termes de mois, constitue également un avantage pour les petites structures. Ceci explique que les grandes entreprises doivent s'appuyer sur des forces externes, qu'elles ne peuvent seules, en interne, faire face à ces défis technologiques et industriels.

Le groupe Thales externalise une grande part de la recherche auparavant réalisée dans son laboratoire central de Corbeville (Orsay), et le groupe Alcatel mise également beau-

\footnotetext{
${ }^{9}$ Seul Thales (ex-Thomson-CSF) a toujours des pratiques positives de partenariat, de travail avec des soustraitants de proximité.

${ }^{10}$ Dans cette étude statistique, la technopole regroupe 80 communes du sud-ouest de l'île-de-France.

${ }^{11}$ L'optique représente en Île-de-France 400 entreprises, 16000 emplois (dont 7000 ingénieurs et techniciens), 1800 chercheurs, 10 organismes de recherche et centres de formation, 1500 publications scientifiques par an, 400 brevets déposés par les entreprises franciliennes et un CA de plus de 5 milliards d'euros. Par ailleurs $55 \%$ des entreprises sont localisées dans la technopole (Essonne et Yvelines) et 17,3\% dans les Hauts-de-Seine, soit $72,4 \%$ dans l'ouest et le sud de la région (source : Opticsvalley.org -2004).
} 
coup sur cette fonction d'interface que peuvent assurer les petites entreprises créées à partir de compétences locales. L'organisation de la recherche en réseau qui s'est développée de façon croissante dans les entreprises depuis les années 80 a été adoptée par deux des acteurs essentiels du pôle optique, l'opérateur de télécommunications, France Télécom, et son équipementier privilégié dans le passé, Alcatel.

En particulier, la création de l'association « Optics Valley », en octobre 1999, par Alcatel, Thales, des PMI de pointe comme Photonetics, et des organismes de recherche comme le CNRS, l'Institut d'optique, l'ENSTA et le GIE Opto+ (pôle de recherche commun créé en 1998 par Alcatel et France Télécom), est représentative du nouveau type de fonctionnement que les grands acteurs cherchent à impulser dans le milieu. Elle s'inscrit dans la nouvelle stratégie de ces groupes, qui veulent saisir les opportunités liées au développement rapide des technologies optiques. Le fait même que l'origine de ces tentatives d'instauration d'un rapport plus étroit avec le milieu local se trouve dans la complexité des nouvelles contraintes de production et d'innovation, constitue un facteur favorable à la profondeur et à la durabilité de ce rapport au territoire.

\section{Le pôle optique : un cluster innovant ou un milieu innovateur?}

L'analyse précédente a montré que ces regroupements d'activités proches par les technologies ou les marchés ne sont pas seulement fondés sur la recherche d'externalités génériques métropolitaines (services, infrastructures, main-d'œuvre qualifiée) mais aussi sur celle d'externalités de milieu (besoin de visibilité ou de contacts, de coopération, d'ambiance, proximité avec la recherche). Si ces activités sont interconnectées entre elles et avec la recherche (réseaux coopératifs d'innovation), et si elles créent des lieux d'intercommunication et d'apprentissage, on se rapproche du concept de milieu innovateur. Si l'agglomération d'activités est essentiellement la recherche de la proximité avec les concurrents et les fournisseurs, ou même les clients, on se rapproche plutôt du concept de cluster. Le deuxième type nous paraît avoir un caractère plus spontané et moins construit, tandis que le premier a un caractère plus volontariste. En tout cas, dans les deux situations, la recherche de la compétitivité grâce à l'innovation est primordiale, même si dans le deuxième cas, elle se fait plutôt par l'émulation et dans le premier par la coopération.

Si, durant les années 80 et 90, les grands acteurs de l'optique se sont concertés pour tenter de résoudre en commun des problèmes technologiques, avec le rôle pivot du IOTA, centre universitaire moteur dans les réseaux d'innovation, les collaborations, dans les années 2000, ont changé d'échelle grâce à l'implication des groupes dans la création de lieux d'interconnexion. Les principaux acteurs décident de créer des structures communes de recherche ou de concertation. Il y a montée en puissance du rôle des groupes et du conseil général de l'Essonne et tentative de coordination et de territorialisation des stratégies ${ }^{12}$. Mais ces actions sont très dépendantes du volontarisme soutenu des acteurs privés, dont le désengagement peut être brutal.

\footnotetext{
${ }^{12}$ Nous nous appuierons pour cette analyse sur les résultats d'une enquête menée auprès des acteurs moteurs du pôle optique (Alcatel, CNRS, Île-de-Science, Institut d'Optique, Syndicat patronal de l'optique, conseil général, « Optics Valley ») (Cohen, Decoster, Tabariés, 2002).
} 


\subsection{Vers un nouveau modèle de localisation et d'apprentissages collectifs}

La dynamique créatrice du territoire se traduit non seulement par une aptitude à réaliser des innovations, mais par le fait que le système productif local acquiert de nouvelles propriétés qui le mettent en mesure d'innover de façon continue (Matteaccioli, 1999). Ce système local d'innovation est alors qualifié de «milieu innovateur » lorsqu'il a une composante organisationnelle (les réseaux d'innovation locaux), une composante cognitive (l'apprentissage collectif) et une composante territoriale d'auto-identification (identité collective). Si les deux premières composantes sont présentes dans le milieu de l'optique, la troisième composante reste très floue, mais surtout elle est certainement à repenser de façon spécifique dans un contexte métropolitain. L'identité collective et la gouvernance locale ne fonctionnent pas dans la métropole selon les mêmes schémas que dans des territoires urbains moins complexes et plus aptes à l'émergence d'un projet de territoire et à la mobilisation d'acteurs porteurs.

\subsubsection{L'émergence d'une logique d'organisation en réseaux}

À côté des relations traditionnelles de marché que les entreprises entretiennent entre elles dans un environnement proche mais surtout lointain, du fait de leur appartenance à une région métropolitaine externalisée, se sont développées des relations hors marché dans le milieu local. Ces dernières regroupent plusieurs formes d'interdépendances : des relations directes entre entreprises et centres de recherche, mais aussi des phénomènes d'incubation à travers l'expérience professionnelle et les savoir-faire contenus dans la culture technique de la technopole, à travers la formation et l'environnement scientifique et technique qu'offrent les universités et les laboratoires universitaires. Des relations plus suivies ont pu s'établir localement, contribuant ainsi à la constitution de réseaux d'innovation à travers lesquels les entreprises peuvent mobiliser les savoir-faire et les pratiques techniques contenus en eux.

Mais cette logique d'organisation en réseaux sur une base locale est le produit d'une lente maturation, aboutissant à un renouvellement fondamental des acteurs : des réseaux impulsés d'en haut par des acteurs étatiques (État, CNRS, ministère de la Recherche) sont de plus en plus impulsés d'en bas par des acteurs locaux (Île-de-Science, Conseil Général).

A. Des réseaux impulsés par des acteurs étatiques, à objectifs généraux, mais aptes à structurer le milieu local

Les actions les plus récentes de mise en réseau sont celles du CNRS et du ministère de la Recherche, qui vise à créer un milieu interactif dans le domaine de l'optique. Le but de «l'Action coordonnée optique » du CNRS, impulsée en 1998 par le directeur de l'Institut d'Optique, est de diffuser l'optique vers des milieux industriels diversifiés, en mettant en relation les industriels et les laboratoires sur des opérations précises, en favorisant la création de groupes de travail entre des chercheurs de culture et de discipline différentes. Tous les fondateurs d' «Optics Valley» se retrouvent dans le comité scientifique de cette «Action coordonnée optique », ce qui est révélateur de la connivence entre les acteurs des technologies optiques, qui a facilité les discussions ultérieures entre les directions d'Alcatel et du CNRS, entre autres.

Même si cette action est du ressort du niveau centralisé de l'État, et reste de l'ordre du global, elle n'est pas, du fait de ses conditions d'émergence, déconnectée des acteurs d'une 
communauté techno-professionnelle, elle repose sur l'engagement de ces acteurs qui vont en être les éléments moteurs ; ces actions contribuent à des objectifs généraux mais aussi locaux dans la mesure où elles ont des effets structurants dans le milieu local, développent les connaissances réciproques et surtout l'émergence de projets. En ce sens elles contribuent à la dynamique de projet et à la construction d'un milieu. On peut d'ailleurs considérer cette «Action» CNRS comme l'un des déclencheurs de ce qui a suivi, à savoir la création des différents réseaux dans l'optique, en particulier d' «Optics Valley », et du projet de plate-forme de transfert technologique.

D'autres actions comme « l'Action Coordonnée Incitative photonique » du ministère de la recherche et la création d'un CNRT (Centre National de Recherche Technologique) de l'optique par ce même ministère sont venues en quelque sorte concurrencer l'action précédente et la création de l'association « Optics Valley », mais comme ces actions s'appuient sur les mêmes acteurs de la communauté optique, ceux-ci ont su organiser les complémentarités entre les actions et dépasser les rivalités entre les deux institutions.

B. Des réseaux impulsés par des acteurs localisés dans le territoire :

d'objectifs généraux vers le soutien aux «clusters »

Un acteur représentatif de ce type de réseaux est l'association « Île-de-Science », qui regroupe 25 établissements de recherche et de formation, publics et privés, situés sur l'ensemble de la technopole. Créée à l'origine (1991) pour coordonner la recherche et la formation et, en particulier, créer des formations communes, elle a élargi le champ de ses actions à la promotion de l'image de la technopole, au soutien à des projets locaux forts comme le synchrotron SOLEIL, et le centre d'échanges scientifiques et technologiques X-Pôle, situé sur le campus de Polytechnique à Palaiseau.

Elle n'a que des moyens incitatifs et son action principale aujourd'hui est de l'ordre de la communication. Son rôle est d'inciter au développement d'interrelations entre les acteurs de recherche et de formation et les industriels, de les installer dans la durée et de contribuer à créer ainsi un « milieu complexe de compétences » (Perrat, 1997).

Ce type d'action de décloisonnement et de mise en réseau, qui s'inscrit dans une perspective relativement générale et globale, peu ciblée, rejoint les actions menées depuis les deux dernières décennies par différentes associations ${ }^{13}$, et se heurte aux forces de résistance et aux inerties des « citadelles » locales. Il ne peut pas faire progresser rapidement la territorialisation des acteurs. Des approches moins globales et basées sur des dynamiques de projet semblent plus aptes à faire évoluer les acteurs vers une construction territoriale.

Le conseil général de l'Essonne en revanche a eu une politique plus ciblée car liée au développement local. Il est passé d'une politique de soutien au transfert à une politique de mise en réseau des partenaires. Il a joué un rôle pionnier en Île-de-France depuis le début des années 90, cherchant à mener une politique globale d'innovation, de valorisation des compétences et des ressources technologiques. Sa politique a visé en quelque sorte à un repositionnement de la recherche, avec la prise en compte, dans l'élaboration de sa politique technologique, des critères de compétitivité du tissu industriel local et de développement des futurs secteurs stratégiques.

\footnotetext{
${ }^{13}$ Cité Scientifique au cours de la décennie 80 essentiellement, associations de transfert telles que les CRITT, ASTER - Île-de-France.
} 
Depuis les élections de 1998, le rôle de celui-ci s'affine et s'articule autour du soutien à la création de «clusters » et de réseaux en particulier dans l'optique et la génomique. Ses actions sont fondées sur des démarches de projet et de mise en réseau : rôle de concertation, de coordination et de fédération des divers acteurs, soutien de projets strictement expertisés et évalués, obtention d'un effet-levier grâce au travail de coordination et au soutien financier.

C'est le modèle d'innovation en réseau qui devient l'objectif et la politique s'élabore à partir du «cluster », qu'il faut au préalable déterminer et visibiliser. Ensuite viennent les politiques de coordination, d'implantation d'entreprises en rapport avec les clusters, de soutien à la recherche quand elle est couplée au développement local (exemple du centre optique dans le cadre de la reconstruction du IOTA, procédure SESAME pour les laboratoires tournés vers les entreprises). En faisant du «portage» de projets, il se veut un des acteurs importants de la mise en réseau des acteurs académiques et industriels, et soutient en particulier l'association « Optics Valley » et sa plate-forme technologique.

La politique des acteurs territoriaux est à resituer dans le cadre de la transformation des méthodes de l'action et du « modèle de localisation français ». Elle relève de cette nouvelle forme d'action collective où prévalent le projet et le contrat, caractérisée par la multiplication «des coalitions autour d'un projet» (Bourdin, 2000). C'est ce nouveau « modèle réflexif de localisation » qui oriente l'action du conseil général de l'Essonne.

\subsubsection{Mise en ouvre d'apprentissages collectifs et stratégie des acteurs nationaux (Alcatel, France Télécom, CNRS)}

Un milieu innovateur est caractérisé par une logique d'apprentissage, c'est-à-dire la capacité des acteurs, constituée au cours du temps, à modifier leur comportement en fonction des transformations de leur environnement. Ces apprentissages portent sur la formation de savoir-faire, le développement de normes de comportement qui permettent de construire un espace de travail commun, la connaissance et la capacité d'identifier en tant qu'opportunité d'interaction les ressources spécifiques des différents acteurs et la relation que les acteurs du milieu entretiennent avec l'environnement externe (Maillat, Quévit, Senn, 1993). On observe depuis peu dans le pôle optique des actions de structuration du milieu et de mise en commun de ressources technologiques initiées par des acteurs nationaux ou locaux, qui mettent en place les conditions favorables à un processus d'apprentissage et d'ancrage territorial.

Les actions les plus importantes concernent Alcatel, France Télécom et le CNRS. En février 2000, Alcatel et le CNRS ont décidé de constituer un pôle de recherche et développement en optoélectronique situé dans le centre de recherche d'Alcatel à Marcoussis. L'évolution des stratégies de recherche de France Télécom et d'Alcatel permet de comprendre comment un processus d'apprentissage a pu démarrer.

France Télécom a entamé en 1998 la réorganisation de sa R\&D pour l'adapter au nouvel environnement de la déréglementation et des NTIC. L'entreprise a rompu avec le modèle linéaire et diffusionniste de la recherche qui fonctionnait bien dans des situations de mâ̂trise des risques techniques et commerciaux et est passée d'une organisation centralisée de sa recherche (le CNET est chargé de la recherche fondamentale pour toute la filière) à un recentrage de la recherche sur son métier d'opérateur en relation avec les unités opérationnelles et instaurant des liens très forts avec les autres acteurs de recherche, académiques et industriels. France Télécom est allé très loin dans cette stratégie, beaucoup plus loin que le 
groupe Alcatel, en externalisant une grande partie de sa recherche (la moitié des résultats de recherche proviennent actuellement des « consortiums » mis en place).

Pour Alcatel, le changement essentiel se situe au milieu des années 90 et concerne le rapprochement de la recherche avec les divisions opérationnelles et la transversalité des projets par rapport aux sites de recherche. Au début des années 90, le centre de recherche d'Alcatel, même s'il était plus lié au marché que celui de Thomson-CSF, était un véritable centre de recherche fondamentale, fonctionnant de manière relativement autonome. Or, aujourd'hui, d'une part, le site majeur de recherche-développement, le Corporate Research Center (CRC) de Marcoussis (60\% de la recherche d'Alcatel) tend à externaliser la recherche fondamentale pure, et d'autre part la stratégie du groupe, la concurrence, les marchés, concourent à la définition des programmes. C'est une nouvelle logique de la recherche industrielle qui se met en place : elle n'est plus un moyen de veille technique mais « est partie intégrante d'une stratégie d'innovation qu'elle contribue à soutenir mais de laquelle elle reçoit également ses axes d'orientation » (Encaoua, Foray, Hatchuel, Mairesse, 2001, p. 37).

Plus que d'externalisation de la recherche, c'est d'une stratégie d'endogénéisation des compétences externes qu'il faut parler. Cette stratégie contribue à accroître la logique de coopération au travers de partenariats directs, bilatéraux ou multilatéraux, et à aider au développement de nouvelles compétences (grâce à un fonds de capital-risque propre, Alcatel Ventures, aux « virtual companies » (start-up internes) et à des incubateurs). Même si l'organisation et les objectifs ont changé, l'innovation se fait actuellement toujours très fortement en interne. Les technologies sont conçues en interne à 85-90\% .

Alcatel développe donc une configuration organisationnelle de veille technologique et d'internalisation sélective des ressources externes, qui se traduit par une politique de partenariat, de mobilisation des ressources où qu'elles soient dans le monde, mais sans qu'il n'y ait aucune implication du groupe dans la structuration des ressources externes. Toutefois, pour répondre aux exigences de mobilisation de compétences pointues, de flexibilité et de partage des coûts et des risques, une logique «prédatrice » est largement insuffisante et Alcatel tente d'aller au-delà de cette simple logique d'internalisation sélective des ressources externes. Deux stratégies sont mises en œuvre, l'une concernant les innovations incrémentales, l'autre les innovations de rupture.

Dans le premier cas, la configuration organisationnelle est relativement aisée à mettre en place pour un grand groupe, qui a su faire évoluer l'organisation interne de son potentiel de R\&D et mettre au point des accords-cadres de partenariat avec des laboratoires, des universités, même si une telle transformation a constitué un profond bouleversement du mode de fonctionnement de la R\&D du groupe et a entraîné une complexité croissante. Les difficultés de couplage entre recherche fondamentale et recherche appliquée, et entre disciplines différentes, ont porté Alcatel à s'impliquer dans la structuration de ces interrelations, pour en accroître l'efficacité et la durabilité, avec la mise en place sur le site principal de recherche de Marcoussis de plusieurs dispositifs.

Quant aux innovations de rupture, elles nécessitent de passer à un seuil organisationnel supérieur et de participer à une structuration plus forte du milieu; la nature même des « externalités technologiques », leur caractère latent et potentiel expliquent que le groupe tente de se connecter de façon durable sur un «milieu complexe de compétences », de passer à une logique d'intervention dans la construction des ressources, afin d'assurer cette mise en continuité des ressources internes et externes. 
Dans le domaine de l'optique, deux acteurs sont importants : le CRC de Marcoussis et la division Alcatel Optronics basée à Nozay (près de Marcoussis), filiale à $100 \%$ du groupe et créée en 1994. Auparavant, les différents centres de recherche en opto-électronique d'Alcatel étaient en quelque sorte en compétition entre eux, dans une logique quasiacadémique, et avec les laboratoires du CNET et de Thomson-CSF. La prise de contrôle de la politique de recherche par les industriels a constitué une évolution importante, rendue possible grâce à l'effet catalyseur d'une opération (plate-forme technologique commune) menée avec un acteur externe, le CNET de Bagneux.

Dans un contexte de forte croissance, le GIE Opto+, créé en 1998 par France Télécom et Alcatel, a approfondi le partenariat engagé entre l'opérateur et son équipementier quelques années auparavant ; le GIE est un pôle commun de recherche et développement spécialisé dans l'optoélectronique entre le CNET Opto de Bagneux et des équipes du CRC de Stuttgart et de Marcoussis regroupés dans le centre de recherche d'Alcatel à Marcoussis. La recherche d'Opto+ est totalement orientée vers les applications et couvre les domaines des composants opto-électroniques et micro-électroniques pour les télécommunications optiques. Du fait d'intérêts communs entre les deux partenaires, on peut parler d'un processus d' « endogénéisation continue » des ressources (Perrat, 1997).

Ce pôle a été renforcé en 2002 par la création d'un nouveau laboratoire par le CNRS, le Laboratoire de Photonique et de Nanostructures, qui a regroupé au CRC de Marcoussis une centaine de personnes issues du CNET et du CNRS. La politique de partenariat renforcé d'Alcatel avec le monde académique l'a ainsi amené à accueillir dans ses locaux de Marcoussis ces équipes du CNRS et à leur offrir de puissants moyens de recherche. Avec ce type de création institutionnelle, on se situe plutôt dans une logique d' "endogénéisation continue mais temporaire », où les relations restent très formelles et la relation au territoire secondaire (Perrat, 1997).

Ces dispositifs illustrent une gestion différente par Alcatel du rapport d'externalité, le passage d'une logique d'internalisation à une logique d'endogénéisation (Catin, 1985). Les adaptations se situent plutôt dans le registre de la logique d' «endogénéisation discrète » avec l'acteur académique et dans celui de l'«endogénéisation continue » (Perrat, 1997) avec l'opérateur industriel France Télécom qui s'est engagé dans un processus plus fusionnel du fait d'intérêts communs peu contradictoires avec Alcatel. L'engagement d'Alcatel dans «Optics Valley » devrait constituer l'étape ultérieure de ce processus.

\subsection{Milieu innovateur ou cluster?}

Un milieu innovateur possède trois propriétés : le fonctionnement en réseaux, la mise en œuvre d'apprentissages collectifs et l'auto-identification. Si les deux premières caractéristiques sont trouvées dans le milieu de l'optique, la troisième semble seulement en émergence. Est-ce dû au fait que le contexte métropolitain empêche cette auto-identification ou plutôt qu'il ne s'agit que d'un cluster, c'est-à-dire un ensemble coordonné d'activités de la même filière, s'organisant plus ou moins sur le plan local ?

\subsubsection{Les stratégies des acteurs territoriaux et nationaux en interaction: \\ "Optics Valley », un pas décisif vers l'auto-identification des acteurs?}

Phénomène caractéristique des milieux innovateurs, les acteurs issus de grandes entreprises, d'institutions de recherche publique et des PME de pointe sont de plus en plus en 
phase dans la mise en œuvre de processus d'apprentissages collectifs, grâce en partie à la mise en place de lieux d'interconnexion renouvelés (Institut d'Optique) ou complètement nouveaux («Optics Valley »), favorisant l'interconnaissance des acteurs.

D'une part l'Institut d'Optique met en place des dispositifs plus ouverts. Il a un rôle pivot en matière de partenariats public/privé grâce à la présence en son sein d'un laboratoire de service, d'équipes du CNRS, traitant à la fois la recherche fondamentale et les applications qui en découlent et collaborant avec toutes sortes de laboratoires et d'entreprises, dont beaucoup sont locaux, et enfin de la Société Française d'Optique, société savante qui réfléchit sur les questions nouvelles qui se posent à la recherche et à l'innovation.

L'Institut d'Optique cherche d'une part à développer ses formations en partenariat avec les industriels du fait de la pénurie d'optroniciens formés en France et d'autre part à favoriser la création de technologie grâce une nouvelle plate-forme technologique de transfert (IOTech) dont le but est d'aider les industriels à externaliser leur recherche amont. Elle devrait fonctionner en complémentarité avec la plateforme mise en place par «Optics Valley », spécialisée dans l'analyse des matériaux, la caractérisation des composants et systèmes et l'expertise ${ }^{14}$. Par ailleurs son laboratoire CNRS veut élargir ses domaines de compétences dans les semi-conducteurs et rapprocher sa recherche des milieux industriels en créant un laboratoire commun avec TRT et Polytechnique, spécialisé dans les nanotechnologies optiques.

D'autre part, une innovation institutionnelle majeure pour le processus de territorialisation a sans doute été la création en 1999 de l'association « Optics Valley », dont nous avons déjà parlé. Plusieurs éléments ont expliqué la création d' «Optics Valley » par un noyau dur représentant acteurs industriels et monde académique: grands groupes (Alcatel et Thomson-CSF), PMI de pointe, comité Richelieu, CNRS, Institut d'Optique, ENSTA, GIE Opto+. La conjonction d'industries en bonne santé, de marchés en plein développement, de compétences fortement concentrées en Île-de-France, d'un comportement entrepreneurial nouveau et d'une nouvelle stratégie d'externalisation de la recherche des grandes entreprises, est apparue comme une opportunité exceptionnelle aux acteurs franciliens de l'optique. Dans un contexte de développement mondial rapide, les technologies optiques étaient devenues un élément structurant des technologies de l'information et de la communication et leur potentiel de transversalisation et de diffusion en fait toujours un secteur d'avenir.

Dans un premier temps, «Optics Valley » est conçu comme un moyen de « visibiliser» le pôle optique existant, afin de lui donner une capacité à communiquer efficacement sur les actions d'envergure qu'il veut mener. Ensuite une démarche de projet et de coopération repose sur quelques acteurs clés, des personnalités du monde industriel, ayant une légitimité à la fois dans la communauté professionnelle et dans la communauté scientifique et sur l'ouverture que représente la Société Française d'Optique et ses lieux d'interaction. Les acteurs d' "Optics Valley » partent de deux constats, celui des potentialités offertes par les technologies optiques et celui de l'obsolescence de l'ancien schéma linéaire de l'innovation ; il s'agit de mettre en commun des moyens pour une recherche précompétitive collective, afin de ne pas être rapidement dépassé au niveau mondial. Seul le fonction-

\footnotetext{
${ }^{14}$ Il s'agit de PRISME, Plate-forme Régionale Île-de-France sud de Mesures et d'Expertises, composée de 6 partenaires (Institut d'Optique, Laboratoire national d'essais, Lycée Fresnel, Supélec, Thales et Université Paris sud (Orsay)).
} 
nement interactif permet de saisir les opportunités de marché et d'y développer des niches, grâce entre autres à la création de start up, ce à quoi se sont ralliés des groupes comme Alcatel et Thales.

Tant sa structure souple que sa plateforme technologique doivent permettre à « Optics Valley » d'optimiser les contacts et les coopérations entre les différents acteurs, et ainsi d'augmenter les occasions d'apprentissage dans le milieu. Les acteurs «nationaux » ont ainsi misé sur le territoire, en favorisant de différentes manières leurs interrelations avec les acteurs plus territorialisés (PMI, laboratoires et écoles) et en permettant à la connaissance de circuler plus vite au sein du milieu de l'optique ${ }^{15}$.

La démarche d' «Optics Valley » se veut radicalement différente des précédentes qui partent d'idées générales : ici c'est le marché, des opportunités et des idées précises qui déclenchent les mobilisations et les interactions. Les projets d' «Optics Valley », dont la plateforme de transfert, ont été soutenus par la région et le département, permettant d'inscrire le pôle optique dans une structuration régionale et de lui donner des moyens importants. Cette démarche s'inscrit donc dans un processus de territorialisation de l'innovation et de constitution d'un milieu innovateur. Cependant, le processus d'auto-identification est loin d'être achevé et risque d'être remis en cause si les grands acteurs se désengageaient.

\subsubsection{Un impact d' "Optics Valley » difficile à évaluer dans une conjoncture difficile pour les télécoms et pour Alcatel}

$\mathrm{Si}$ «Optics Valley » est de nature à visibiliser le pôle optique pour l'extérieur, il peut aussi aider à construire l'identité du pôle pour l'ensemble des acteurs y participant, en créant une communauté de destin entre les différents partenaires qui devrait reposer de plus en plus sur le partage de valeurs et de conceptions communes aussi bien professionnelles que sociales.

Toutefois la question essentielle qui reste encore ouverte à l'heure actuelle est celle du sens de ce processus pour certains acteurs. L'engagement, actif et même moteur, d'Alcatel dans « Optics Valley » n'est-il qu'une simple tentative d'amélioration des mises en synergie d'équipes de recherche, un approfondissement du «milieu complexe de compétences », ou plus fondamentalement le groupe s'engage-t-il dans un processus de «création institutionnelle localisante » (Perrat, 1997) ? Et plus grave encore, les fortes turbulences observées depuis 2001 dans le monde des télécoms et des composants optiques en particulier obligent à reposer la question de la territorialisation des grandes entreprises. Alcatel, qui est le pivot du regroupement autour de l'optique, a vu ses marchés s'effondrer et a dû céder son usine de Nozay (Alcatel Optronics) à un groupe américain. Si la masse critique de compétences rassemblées en Essonne ne peut pas disparaître, des réorientations de recherche peuvent être faites et surtout la création de nouvelles start up en optique pourrait ne pas se réaliser, ce qui entraînerait un affaiblissement du milieu. Les difficultés d'Alcatel pourraient remettre en cause ce choix stratégique de l'optronique fait dans les années 90 ainsi que son implication dans le territoire, à moins qu'au contraire elles ne le poussent à renforcer ses liens avec la recherche publique, pour tenter d'investir sur le long

\footnotetext{
15 Parmi les actions récentes, citons l'organisation à l'université d'Orsay d'un atelier sur la « biophotonique » avec Genopole, dans le but de définir les segments de la biophotonique porteurs de développement et de transfert, préfigurant un couplage des deux clusters de l'Essonne.
} 
terme. De plus les acteurs publics, comme l'État, la Région, le département et le CNRS ont une capacité d'empêcher la déstructuration de cette filière ou de ce milieu par des politiques ciblées, comme ils l'ont déjà fait par le passé et comme ils continuent de le faire ${ }^{16}$. Enfin les technologies optiques restent des technologies d'avenir avec nombre de marchés et applications différents, en dehors des télécoms (opto-électronique, bio-photonique, optique aéronautique, optique mécanique, automobile).

Malgré l'incertitude qui pèse sur la capacité du pôle optique à faire émerger en son sein un authentique sentiment d'appartenance de l'ensemble des acteurs à leur milieu local, on a pu observer une progressive construction d'une dynamique territoriale à travers l'émergence de logiques réticulaires et cognitives. Le degré d'organisation du milieu de l'optique dépasse la simple agglomération d'activités interconnectées, le cluster, pour se rapprocher d'un type de milieu innovateur métropolitain, qui serait plus extraverti, du fait du poids des grandes entreprises présentes et de leurs stratégies en perpétuelle reconfiguration, et du rôle de l'État et de ses émanations (CEA, Universités, CNRS, France Télécom...), et qui aurait un rapport au territoire plus lâche. Dans ce milieu, les acteurs de formation et de recherche sont les pivots du processus de territorialisation, et la réalisation de lieux d'interconnexion forts entre privé et public tendra à favoriser la diffusion de la connaissance dans les deux sens, du marché à la technologie et de la technologie au marché.

\section{Conclusion}

L'observation pendant plusieurs années d'une zone en plein développement, l'Île-deFrance sud, nous a permis de montrer qu'à la suite de décisions étatiques de délocaliser ou de créer de grandes institutions de recherche, il s'est produit d'abord un phénomène de desserrement, puis de polarisation autour des filières de haute technologie liées aux grands centres de recherche finalisés. Puis, dans le cadre d'une nouvelle politique visant à multiplier les relations entre la recherche et l'industrie, se sont constitués peu à peu des réseaux d'entreprises autour de technologies. La complexification des réseaux de relations dans la zone technopolitaine entre recherche, enseignement, groupes et PMI, dans le secteur de l'optique en particulier, a amené à se poser la question de la territorialisation des dynamiques d'innovation. Ressortissent-elles à la logique de la filière ou du cluster, où l'organisation se réalise de façon spontanée, ou plutôt à celle du milieu innovateur, où l'innovation est organisée et épaulée par les différents moyens que se donnent les acteurs (réseaux de coopération, lieux d'apprentissage et d'intercommunication) sur le long terme?

La présence de nombreux réseaux d'innovation, la constitution de lieux d'interconnexion où peuvent se réaliser des processus d'apprentissage élaborés, ainsi que la volonté de donner une identité au pôle ainsi constitué, grâce à la création d'une association regroupant l'ensemble des acteurs et soutenue par les collectivités régionale et locale, nous permettent de qualifier ce milieu d'innovateur. En effet, l'organisation du milieu a pour but principal l'innovation. Malgré la plus grande fragilité de ce type de milieu, liée au poids des grandes entreprises et de leur logique dominante d'extraversion par rapport au tissu

\footnotetext{
${ }^{16}$ Par exemple attribution de bourses de recherche post-doctorales par la Région en vue de favoriser la coopération entre laboratoires publics et entreprises d'optique franciliennes via l'intégration de jeunes chercheurs.
} 
local, il nous semble que ce milieu est parvenu à un niveau d'organisation supérieur, qui lui permet d'assurer une certaine forme de pérennité sur le long terme, pas seulement grâce aux externalités métropolitaines que lui fournit sa présence dans une région-capitale, mais aussi grâce aux externalités technopolitaines ou de « milieu » qui se sont construites sur le long terme. En effet, l'intérêt de la territorialisation des compétences, y compris par des grands groupes, est de rendre relativement improbable, du moins à moyen terme, les comportements « opportunistes », du fait de la densité des réseaux créés, tant pour la recherche et l'innovation que pour la formation, qui rendrait leur perte coûteuse pour les entreprises y ayant investi.

\section{Bibliographie}

Aglietta M., Boyer R.,1983, Pôles de compétitivité, stratégie industrielle et politique macro-économique, CEPREMAP.

Amendola M., 1985, Specific Labour Inputs and the Process of Innovation, Communication to the Conference Structural Change and Technical Progress, Manchester.

Aydalot P., 1985, Economie régionale et urbaine, Economica, Paris.

Aydalot P., 1986a, Les technologies nouvelles et les formes actuelles de la division spatiale du travail, Cahiers du C $3 E 47$.

Aydalot P., 1986b, L'aptitude des milieux locaux à promouvoir l'innovation technologique, in Federwisch J. et Zoller H. (éds), Technologies nouvelles et développement régional, Economica, Paris, 41-58.

Aydalot P., 1986c, Présentation, in Aydalot P. (éd.), Milieux innovateurs en Europe, Gremi, Paris, 9-14.

Beckouche P., Cohen J., Damette F., Fischer J-Ch. et Scheibling J., 1989, Métropolisation et aires métropolitaines : internationalisation et enjeu urbain, Paris, Rapport pour le Commissariat général du Plan dans le cadre du GIP « Mutations économiques et urbanisation ».

Belis-Bergouignan M.-C., Bordevave G. et Lung Y., 1993, Le mirage de la territorialisation de la grande entreprise. Le redéploiement spatial de Ford, Revue d'Économie Régionale et Urbaine 3, 527-544.

Blanc C., 2004, Pour un éco-système de la croissance, rapport au Premier Ministre, la Documentation Française

Bourdin A., 2000, La question locale, PUF, Paris.

Callon M., Laredo P., 1990, L'impact des programmes communautaires sur le tissu scientifique et technique français, rapport du Centre de Sociologie de l'Innovation.

Castells M., 1996, The rise of Network Society, Blackwell Publishers, Oxford, et traduction française 1999 La société en réseaux - l'ère de l'information, Fayard, Paris.

Catin M., 1985, Effets externes, marché et systèmes de décision collective, Cujas, Paris.

Cohen E., 2002, De l'État dirigiste à l'État régulateur, Sciences humaines 133, 38-41.

Cohen J., Decoster E., Tabariés M., 2001, Paris : urban area, technopolitan spaces and innovative firms, the dynamics of innovation, in Simmie J. (dir.), Innovatives Cities, Spon Press, London and New York, 157-190.

Cohen J., Decoster E., Tabariés M., 2002, De l'atome au photon, ou d'une dynamique de « complexe militaroindustriel et nucléaire » vers une dynamique de "cluster», rapport de recherche CNRS, www.univ-mlv.fr/ $\sim$ Www-ltmu/decoster.htm

Colletis G., Gilly J.-P., Pecqueur B., Perrat J., Zimmerman J.-B., 1997, Firmes et territoires : entre nomadisme et ancrage, Espaces et Sociétés 88-89, 115-137.

Decoster E., 1991, Les phénomènes de technopolisation en Ile-de-France sud : les collaborations rechercheIndustrie, Cahiers du GDR 874 du CNRS 8.

Decoster E., Matteaccioli A., Peyrache V., Tabariés M., 1993, Les réseaux d'innovation en région parisienne : micro-milieux en émergence, in Maillat D., Quévit M., Senn L. (éds), Réseaux d'innovation et milieux innovateurs : un pari pour le développement régional, GREMI, EDES, Neuchâtel, 149-179.

Decoster E., Tabariés M., 1992, Le développement technopolitain : le pôle Saclay-Massy, nouveau territoire, nouveaux pouvoirs, nouvelle organisation économique ?, Cahiers du GDR 874 du CNRS 10, 75-104.

Encaoua D., Foray D., Hatchuel A., Mairesse J., 2001, Les enjeux économiques de l'innovation, Bilan scientifique du programme CNRS. 
Gaffard J.-L., 1986, Restructuration de l'espace économique et trajectoires technologiques, in Aydalot P. (éd.), Milieux innovateurs en Europe, GREMI, 17-27.

Hägerstrand, 1967, Innovation Diffusion as a Spatial Process, Chicago.

Maillat D., Quévit M., Senn L.,1993, Réseaux d'innovation et milieux innovateurs, in Maillat D., Quévit M., Senn L. (éds), Réseaux d'innovation et milieux innovateurs : un pari pour le développement régional, GREMI, EDES, Neuchâtel, 3-12.

Matteaccioli A, 1999, Auto-organisation et émergence des milieux innovateurs, Revue d'Économie Régionale et Urbaine 3, 489-511.

Matteaccioli A., 2004, Philippe Aydalot, pionnier de l'économie territoriale, L'Harmattan, Paris.

Markusen A., 1985, Profit cycles, oligopoly and régional development, MIT Press, Cambridge, Massachussets.

Perrat J. 1997, Une clé de lecture du rapport firmes/territoires : la notion d'externalité, Espaces et Sociétés 88-89, 207-236.

Perroux F., 1964, L'Économie du XX $X^{e}$ siècle, PUF, Paris.

Perroux F., 1993, Euvres complètes tome VI - Théorie et histoire de la pensée économique-Marx, Schumpeter,

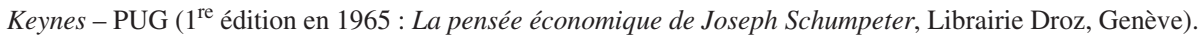

Porter M. E., 1998, Clusters and the new economics of competition, Harvard Business Review, novembredécembre.

Porter M. E., 2000, Location, competition and économic development: local clusters in a global economy, Economic development quarterly, volume 14, 1, 15-34.

Pred, 1966, The Spatial Dynamics of U.S. Urban industrial growth : 1800-1914, Cambridge, Massachussets.

Quévit M., Van Doren P., 1997, The Problem of Innovative Milieux and Territorial Structural Adjustment Policies, in Ratti R., Bramanti A., Gordon R. (eds), The Dynamics of Innovative Regions, Ashgate, 343-365.

Rallet A., 1993, Choix de proximité et processus d'innovation technologique, Revue d'Économie Régionale et Urbaine 3, 365-386.

Rogers E. M., Shoemaker F.F., 1971, Communication of Innovations : A Cross-Cultural Approach, 2d. Ed. New York, London.

Sassen S., 1991, The global city: New York, London, Tokyo, Princeton, University Press, traduction française 1996, La ville globale : New York, Londres, Tokyo, Descartes, Paris.

Schmookler J., 1966, Invention and Economic Growth, Harvard University Press, Cambridge.

Serfati C., 1994, Les interrelations entre l'innovation militaire et la politique technologique en France, communication au colloque Performances technologiques et performances économiques, GREGI, BETA, CERETIM, 14 octobre, Le Mans.

Veltz P., 1993, Logiques d'entreprises et territoires : les nouvelles règles du jeu, in Veltz P. et Savy M. (éds) Les nouveaux espaces de l'entreprise, DATAR, Éditions de l'Aube, 47-79.

Veltz P., 1999, Territoires innovateurs : de quelle innovation parle-t-on ?, Revue d'Économie Régionale et Urbaine 3, 607-616.

Vernon, 1966, International investment and internationalisation in the product cycle, Quaterly Journal of Economics, 80.

\section{Annexes}

Liste des six enquêtes menées par l'équipe: GREMI 1 sur les facteurs d'innovation locaux et les trajectoires d'innovation des entreprises (1985), GREMI 2 sur les liens créés au cours du processus d'innovation (1987), l'enquête sur la création d'entreprise (1987), GREMI 3 sur les réseaux d'innovation et les interrelations réseaux-milieu (1990), l'enquête sur les collaborations des entreprises innovant dans des réseaux BRITE-Euram (2000) et l'enquête sur les stratégies récentes des acteurs du pôle optique (2001). 


\section{Sigles}

AES : Association des Établissements Scientifiques

ASTER Ile-de-France : Appui scientifique et Technique aux Entreprises Régionales

BRITE : Basic Research for Industrial Technologies for Europe

CEA : Commissariat à l'Énergie Atomique

CEETAM : Centre d'Études et d'Enseignement des Technologies Appliquées aux Micro et nano-structures

CEN : Centre d'Études Nucléaires

CNES : Centre National d'Études Spatiales

CNET : Centre National d'Étude des Télécommunications

CNRS : Centre National de la Recherche Scientifique

CNRT : Centre National de Recherche Technologique

CRC : Corporate Research Center (Centre de recherche d'Alcatel)

CRITT : Centre Régional d'innovation et de transfert technologique

DEA : Diplôme d'Études Approfondies

ENSIA : École Nationale Supérieur des Industries Agroalimentaires

ENS : École nationale supérieure

ENSTA : École Nationale Supérieure des Techniques Avancées

GIE : Groupement d'Intérêt Économique

GIFO : Groupement des industries françaises de l'optique

IOTA : Institut d'Optique Théorique et Appliquée

IUT : Institut Universitaire de Technologie

SESAME : Soutien aux Équipes Scientifiques pour l'Acquisition de Moyens Expérimentaux

TRT : Thales Research and Technology France (Centre de recherche de Thales) 


\section{Cartes}

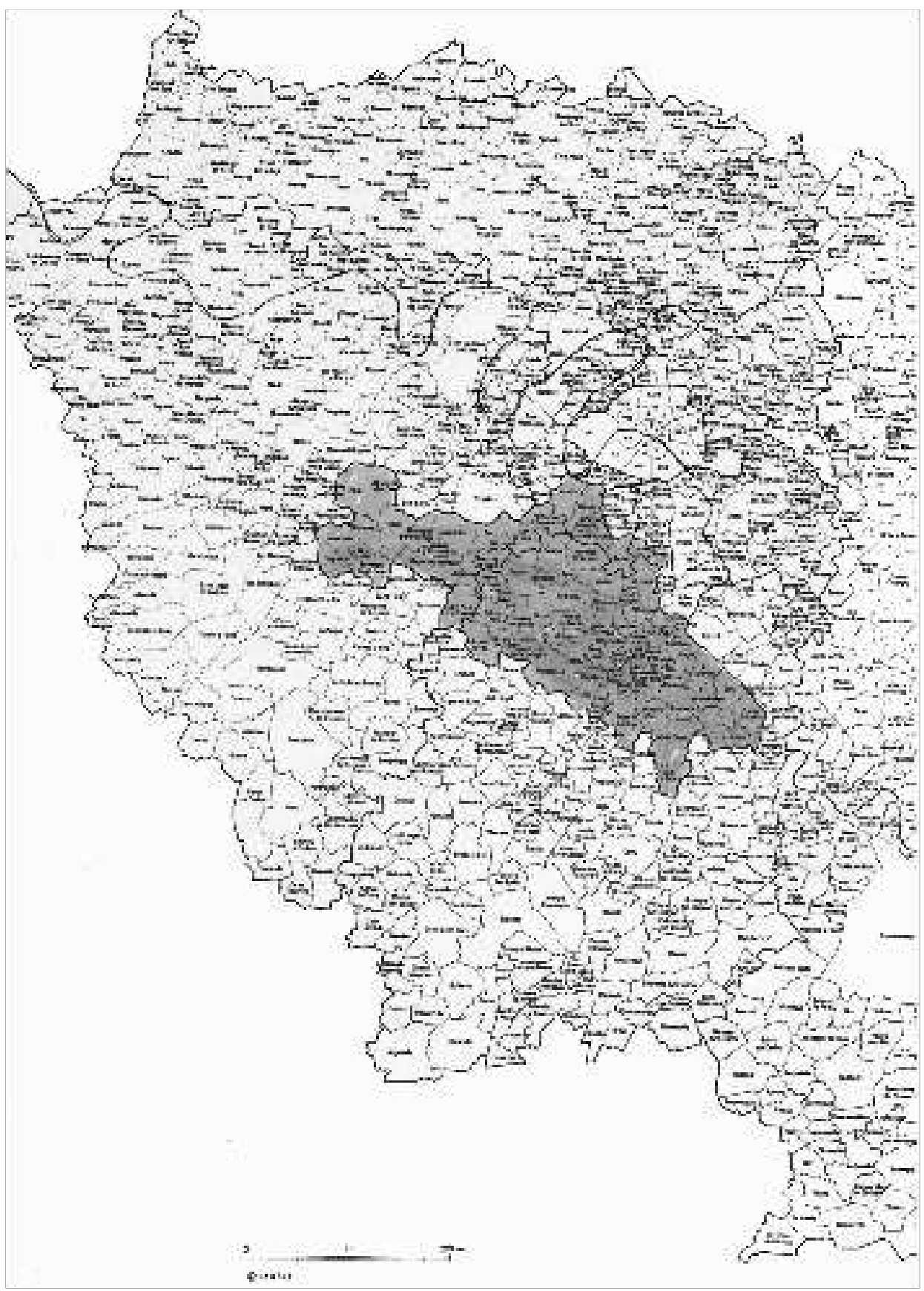

Carte 1. Les quatre-vingts communes de l'étude. 


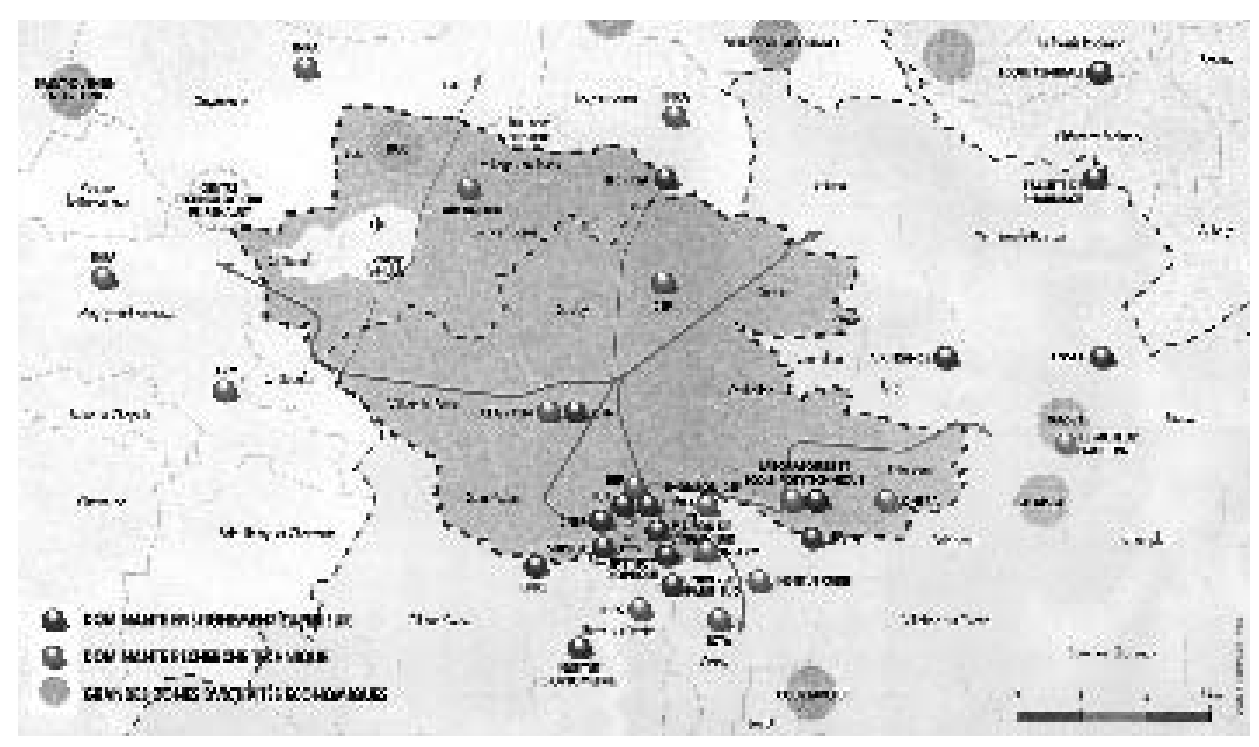

Source : District du plateau de Saclay 1995

Carte 2. Les principales localisations scientifiques et économiques autour du plateau de Saclay. 